\title{
La simulación y disimulación en medicina evaluadora
}

\section{Simulation and deception in assessing practice}

\section{César Eloy Díaz Salazar}

Médico Inspector del Cuerpo de Inspección Sanitaria de la Administración de la Seguridad Social; Unidad Médica del Equipo de Valoración de Incapacidades; Dirección Provincial de Instituto Nacional de la Seguridad Social. Sevilla. España

\section{Recibido: 05-06-14}

Aceptado: 23-06-14

\section{Correspondencia}

César Eloy Díaz Salazar.

Médico Inspector del Cuerpo de Inspección Sanitaria de la Administración de la Seguridad

Social; Unidad Médica del Equipo de Valoración de Incapacidades; Dirección Provincial

de Instituto Nacional de la Seguridad Social. Sevilla. España

Correo electrónico: cesar-eloy.diaz@seg-social.es

Resumen

Cuando un paciente acude al médico asistencial, su principal interés es, en general, ser curado de su enfermedad; pero en la medicina evaluadora suelen estar en juego otros intereses muy distintos (principalmente económicos, aunque también otro tipo de prestaciones sociales, por ejemplo), por lo que no es infrecuente que el paciente, o sujeto objeto de la valoración, intente obtener el mayor beneficio posible de su situación, incluso utilizando todo tipo de artimañas (exageración, simulación, disimulación, etc.) para intentar engañar al médico evaluador sobre la gravedad o afectación funcional que le produce la patología que presenta o alega.

Esto no es algo nuevo, y podemos encontrar numerosos antecedentes a lo largo de la Historia, tanto religiosa (Biblia, por ejemplo), como civil (libros de historia griegos, literatura, etc.) y médica (Hipócrates, Galeno, etc.).

La finalidad de la simulación es siempre la misma, y algunos de los métodos empleados también, pero también han ido desarrollándose diversas técnicas para lograrla y, por ende, también para detectarla.

Diversos autores médicos han intentado describir los indicadores que pueden orientar hacia la existencia de algún tipo de simulación, han establecido técnicas para intentar llegar a su diagnóstico y diferenciación de la afectación real, y han descrito las distintas formas o modalidades en que se puede presentar.

Con este artículo se intenta "encender" en la conciencia del médico valorador "la bombilla" que le haga pensar en su existencia, y señalarle algunas herramientas que le pueden ser útiles en su quehacer diario.

Med Segur Trab (Internet) 2014; 60 (235) 379-391

Palabras clave: Simulación, exageración, criterios, modalidades.

Abstract

When a patient goes to the welfare doctor, its main interest is, in general, cured being of its disease; but in the evaluator medicine usually they are in game other very different interests (mainly economic, although 
also another type of social benefits, for example), reason why is not infrequent that the patient, or subject object of the valuation, tries to obtain the greater possible benefit of his situation, even using all type of ruses (exaggeration, simulation, dissimulation, etc.) to try to deceive the evaluator doctor on the gravity or functional affectation that produces the pathology to him that presents/displays or alleges. This is not something new, and we can find numerous antecedents throughout History, as much religious (Bible, for example), like civilian (Greek books of history, Literature, etc.) and medical (Hippocrates, Galen, etc.). The purpose of the simulation is always the same one, and some of the methods also used, but also they have been developed diverse techniques to obtain it and, therefore, also to detect it. Diverse medical authors have tried to describe the indicators that can orient towards the existence of some type of simulation, they have established technical to try to arrive at his diagnosis and differentiation from the real affectation, and have described to the different forms or modalities in which it is possible to be presented/displayed. With this article it is tried "to ignite" in the conscience of the evaluator doctor "the light bulb" that makes think him about its existence, and to indicate some tools to him that him can be useful in their daily task.

Med Segur Trab (Internet) 2014; 60 (235) 379-391

Key words: simulation, exaggeration, criteria, modalities. 


\section{INTRODUCCIÓN}

En general, todos tenemos claro en la conciencia que en medicina asistencial el enfermo acude al médico con el fin de curar su patología, y que él es el primer interesado en ser sincero con su médico.

Por el contrario, y de forma bastante frecuente, en medicina evaluadora no ocurre esto, ya que hay otros intereses muy diversos del paciente (beneficios económicos, revancha, odio, etc.), y estos hacen, muchas veces, que la persona no actúe de forma tan sincera como sería de esperar; y puede hacerlo mintiendo, simulando o disimulando, con la última intención de engañar al médico evaluador por medio de esas artimañas.

Así, en medicina evaluadora, como en el resto de la Medicina, no basta con saber la clínica y dominar el diagnóstico, sino que también hay que saber descubrir todas estas formas de superchería y falsedad.

Este interés existente en medicina evaluadora por la simulación y disimulación de patologías dentro de la valoración del daño corporal no es algo reciente, sino que se remonta a los tiempos más antiguos conocidos; lo que sí es novedoso es que, ahora, dicho interés ha aumentado, y lo hace cada día, debido a las importantes repercusiones económicas y sociales que estas situaciones conllevan.

\section{CONCEPTO Y DEFINICIÓN DE SIMULACIÓN}

Para poder definir la simulación hay que tener en cuenta, en primer lugar, que "todos los hombres son más o menos simuladores, aunque sólo en algunos la simulación es el medio habitual y preferente de lucha por la vida" (Huertas, 1991), y también que "la simulación es para el hombre una necesidad de adaptación al medio" (Porot, 1921).

Como se adelantaba en la introducción, y siguiendo a Simonín y Estarellas ${ }^{1}$ (1997), se trata de un fraude consciente y razonado que consiste en provocar, imitar o exagerar trastornos mórbidos subjetivos u objetivos con un fin interesado.

Desde el punto de la Psicología, es equivalente al engaño, aunque debe darse con una expresión verbal o apreciable de una "inverdad", requiriendo una expresión psicomotora; es decir, es una mentira plástica, por lo que es imprescindible la ejecución de ciertos actos o la reproducción de algunas actividades que carezcan de motivación real. Siempre está encaminada a conseguir diversos fines o ventajas personales a las que no se tendría acceso sin el fraude.

También puede definirse, como refiere Presentación Blasco ${ }^{2}$, como un proceso psíquico caracterizado por la decisión consciente de reproducir trastornos patológicos valiéndose de la imitación más o menos directa, con la intención de engañar a alguien, manteniendo el engaño con ayuda de un esfuerzo continuo durante un tiempo más o menos prolongado (según Minkowski).

Según Gisbert Calabuig ${ }^{3}$, la simulación presenta las siguientes características:

1. Voluntariedad consciente del fraude.

2. Imitación de trastornos patológicos o de sus síntomas.

3. Finalidad utilitaria: beneficio o provecho inmediato para el simulador.

Según la CIE 10, debe existir "la ausencia de enfermedad".

Y el diccionario de la Real Academia de la Lengua Española reza que simular es representar una cosa, fingiendo o imitando lo que no es, y disimular es ocultar algo que uno siente o padece.

También "disimulación” significa "arte con que se oculta lo que se siente o se sabe". 
La palabra simulación deriva de la latina simulatio (acción de simular o imitar lo que no se es).

De forma similar, podemos decir que la simulación es un fraude o engaño consistente en aparentar, producir o exagerar signos o síntomas patológicos, síndromes e incluso enfermedades, con una intención utilitaria para el simulador. Es, por tanto, una manifestación conscientemente falsa, cuyo fin es engañar a otros. O dicho de otro modo, la simulación es la producción voluntaria de síntomas físicos o psíquicos falsos o groseramente exagerados, con el fin de obtener un beneficio secundario ${ }^{4}$.

Por el contrario, pero en el mismo sentido, disimular una enfermedad o lesión es el hecho consciente de encubrirla total o parcialmente con una finalidad utilitaria.

La simulación y el fraude asociado tienen consecuencias en la economía general, en la colectividad, al tener que soportar cargas injustificadas y potenciar "la cultura del pensionismo". Por ello, incluso aunque el sujeto no consiga lo que quiere, simplemente con sus acciones, va a ocasionar unos gastos sociales (gastos de fraude) ${ }^{5}$, que recaen sobre la sociedad en general y sobre diversas Instituciones en particular (tabla 2).

Gastos de fraude:

- Médicos y paramédicos.

- Farmacéuticos.

- Hospitalarios y de diagnóstico.

- De incapacidad temporal.

- De secuelas permanentes.

- Laborales de descenso de la producción.

- Laborales de sustituciones, convenios, cotizaciones dobles, etc.

- Otros.

Instituciones que soportan estos gastos:

- Hacienda.

- Seguridad Social.

- Compañías aseguradoras.

- Mutuas de accidentes.

- Empresas.

- Otras.

\section{ANTECEDENTES HISTÓRICOS DE LA SIMULACIÓN}

La simulación es un tipo de conducta humana tan antigua como la humanidad, y tanto datos históricos (de Grecia, Roma, la Edad Media, etc.) como textos sagrados (la Biblia) nos muestran que ha existido en todas las épocas, en todas las civilizaciones y en todas las clases sociales.

Se ha indicado que sus pilares son "la mentira, el egoísmo y la hipocresía", y éstos parecen ser inherentes a la condición humana.

Decía Pascal que "el hombre no es más que disfraz, mentira e hipocresía, y todas estas disposiciones tan alejadas de la justicia y de la razón tienen una raíz natural en su corazón”.

Incluso podemos decir que la simulación es más antigua que el hombre mismo, ya que incluso los animales, de las formas más diversas, se sirven del disfraz, del disimulo y del mimetismo o camuflaje para defenderse de sus depredadores (muchos animales, grandes y pequeños, "se hacen los muertos" para huir de sus perseguidores y utilizan otras artimañas para engañar y hacer caer en la trampa a sus presas o pasar desapercibidos) (J.M. Reverte). 
En la Biblia se recogen claros casos de simulación de enfermedades:

- Raquel, hija de Labán, simula una menstruación para que no se descubra su robo ("no se enoje mi señor, porque no me puedo levantar delante de ti, pues estoy con la costumbre de las mujeres").

- El rey David, al ser descubierto en su refugio en casa del rey Aquis, "se fingió loco entre ellos, comenzó a demudar su semblante, y se dejaba caer entre los brazos de la gente, dándose de cabezazos contra las puertas y haciendo correr la saliva por su barba".

- Jacob simula, con la ayuda de su madre Rebeca, ser su velludo hermano Esaú con unas pieles de cabrito para que su padre, Isaac, ya viejo y ciego, lo bendiga y le entregue la primogenitura (simulación, sustitución o suplantación de personalidad).

- Amnón, hijo de David, enamorado de su propia hermana, simula estar enfermo para abusar de ella.

A lo largo de la historia muchos autores (médicos, historiadores, escritores, etc.) han tratado sobre este concepto:

- Hipócrates, en su Tratado de los aires, de las aguas y de los lugares, describió de una forma científica la simulación frecuente en los soldados de los ejércitos asiáticos para evitar luchar en las guerras.

- Galeno estableció unas reglas para diferenciar los dolores reales de los simulados, dando lugar al primer tratado sobre las enfermedades simuladas.

- En una ocasión, Ulises, para no ir a la guerra de Troya y quedarse con Penélope, se fingió loco poniendo un buey y un caballo a arar y sembrando sal.

- Suetonio cuenta que Claudio, cuando llegó a ser emperador, contó que había simulado ser imbécil para salvar la vida durante el reinado de Calígula.

- Ejemplos en la picaresca española, en todos nuestros grandes escritores.

- Sixto V simuló una vejez precoz, y cuando lo nombraron Papa, tiró las muletas, se irguió y entonó el Te Deum con voz potente.

- En los inicios de la medicina legal clásica, Ambrosio Paré, Fortunatus Fidelis, Silvaticus y Zacchias realizaron estudios sobre el tema.

- En nuestro medio, Cristóbal Pérez Herrera, médico de galeras en el siglo XVI, publicó sobre la simulación.

Y su máxima extensión y desarrollo se produce a partir de la aplicación de las leyes sociales de protección ante accidentes de trabajo, accidentes de circulación y pensiones (protección social), detectándose este tipo de engaño o mentira ${ }^{6}$ en todas las culturas, clases sociales y edades (niño que finge enfermedad para no ir al colegio, por ejemplo).

\section{CRITERIOS DE SIMULACIÓN}

El DSM-IV-TR ${ }^{2,7}$ está recogida dentro de la codificación "Otros problemas que pueden ser objeto de atención clínica", y se define como la "producción intencionada de síntomas físicos o psicológicos desproporcionados o falsos, activados por incentivos externos como no realizar el servicio militar, evitar un trabajo, obtener una compensación económica, escapar de una condena criminal u obtener drogas”. También se indica que puede considerarse una conducta adaptativa en algunos casos.

Los criterios diagnósticos, se darían si se observa alguna de las combinaciones siguientes ("Índices de sospecha")

- Presentación en un contexto médico-legal.

- Discrepancia acusada entre el estrés o la alteración/lesión explicados/referidos por la persona y los datos objetivos de la exploración médica. 
- Falta de cooperación y/o cumplimentación terapéutica durante la valoración diagnóstica; ausencia de respuesta al tratamiento convencional.

- Presentación de trastornos o rasgos caracteriales antisociales de la personalidad.

Otro autor que ha tratado profundamente del tema es Yudofsky, quien estableció en 1989 los siguientes criterios indicadores de simulación, con alguna leve modificación:

- Datos de historial clínico y exploración no concordantes con las quejas.

- Síntomas mal definidos, erráticos o vagos.

- Dramatización de las quejas.

- Mala colaboración del sujeto en las exploraciones.

- Resistencia a aceptar un diagnóstico favorable.

- Presencia de heridas probablemente autoprovocadas.

- Análisis toxicológicos positivos.

- Manipulación del historial clínico, pruebas u otros registros.

- Historia de frecuentes heridas o accidentes.

- Perspectivas de cobrar una indemnización o pensión.

- Petición de drogas de abuso.

- El trastorno permite evitar actividades o situaciones desagradables.

- El trastorno puede exculpar de responsabilidades penales o del encarcelamiento.

- Existencia de una personalidad antisocial previa.

Samuel y Mittenberg (2006) destacan aquellos aspectos que contribuyen a la sospecha de presencia de simulación en el contexto médico-legal:

- Motivación y circunstancias: incentivos económicos, solución a problemas socioeconómicos, conducta antisocial, insatisfacción profesional, conflictos laborales, situación cercana a la jubilación e historia previa de engaño, simulación o actos deshonestos.

- Sintomatología: presencia de síntomas atípicos o inusuales, exageración de la sintomatología, resultados en los tests psicológicos y sintomatología incongruente con el curso normal de la enfermedad.

- Presentación ante la entrevista: falta de cooperación con el evaluador y con la divulgación de la información y discrepancias entre los datos de la entrevista y la historia clínica o la documentación preexistente.

- Actividad/conducta fuera de la entrevista: actividad laboral durante el período de reclamación, realización de actividades lúdicas, no relacionadas con el trabajo, buen funcionamiento excepto en aquello relacionado con el desempeño de la actividad laboral, falta de compromiso con el tratamiento y estar bajo vigilancia.

Los criterios diagnósticos referidos a la discapacidad relacionada con la simulación del dolor, según Bianchini, Greve y Glynn (2005) son:

- Criterio A.- Evidencia de incentivos externos significativos.

- Criterio B.- Evidencia procedente de la evaluación física:

- Sesgos en el rendimiento físico.

- Discrepancia entre la valoración subjetiva de dolor (EVA, por ejemplo) y la reactividad psicofisiológica concomitante.

- Los síntomas o las quejas informadas por el paciente son sustancialmente divergentes con la evidencia fisopatológica existente.

- Discrepancia entre la actividad física mostrada por el paciente durante la evaluación formal con la actividad física demostrada cuando el individuo no sabe que está siendo evaluado. 
- Criterio C.- Evidencia procedente de la evaluación neuropsicológica:

- Rendimiento por debajo del azar en pruebas de elección forzada que evalúan funciones cognitivas o perceptivas.

- Rendimiento consistente con la exageración de la discapacidad en pruebas que evalúan funcionamiento cognitivo o perceptivo.

- Discrepancias entre los resultados de las pruebas neuropsicológicas y el conocimiento existente del funcionamiento cerebral.

- Discrepancias entre los resultados de las pruebas neuropsicológicas y la conducta observable y objetivable del paciente.

- Criterio D.- Evidencia procedente de la evaluación mediante autoinforme:

- La discrepancia de la conducta del paciente cuando es formalmente evaluado versus cuando no sabe que está siendo evaluado.

- Las quejas autoinformadas no son consistentes con la evolución de la historia clínica del paciente.

- Los síntomas autoinformados por el paciente son discrepantes con los patrones conocidos de funcionamiento fisiológico y neurológico del dolor y la discapacidad referida a la patología en cuestión.

- Los síntomas autoinformados por el paciente son discrepantes con la conducta observable y objetivable.

- Presentación de un patrón de exageración de síntomas en pruebas de personalidad de amplio espectro y pruebas de screening de simulación de síntomas.

- Criterio E.- Los criterios mencionados no se explican mejor por factores psicopatológicos, neurológicos o evolutivos.

También podemos encontrar referencias a diversos indicadores clínicos de sospecha de simulación:

- Existencia de un beneficio externo (prolongación de baja laboral, obtención de indemnizaciones, incapacidad, discapacidad, etc.).

- Discrepancia entre las pruebas médicas objetivas (Rx, RNM, TAC, signos de Waddell, Posturografía, etc.) y la valoración subjetiva del dolor y la discapacidad por parte del paciente.

- Distorsiones de respuesta en las pruebas de autoinforme (MMPI-2, SIMS, etc.).

- Discrepancias entre las pruebas de rendimiento neurocognitivo y la valoración subjetiva de dolor y la discapacidad por parte del paciente.

- Discrepancias entre las conductas de dolor y la valoración subjetiva autoinformada del sufrimiento del paciente.

- Baja adherencia a las prescripciones médicas y el tratamiento médico y/o psicológico.

- Incongruencia entre signos y síntomas presentados con el curso previsible de la patología en cuestión.

- La frecuencia y/o intensidad y/o duración de los síntomas excede con mucho lo usual en la patología diagnosticada.

- Duración del tratamiento muy por encima de lo esperable, sin base etiológica para explicarlo.

- Escasos o nulos avances terapéuticos.

- Recidivas coincidentes con la posibilidad de alta laboral.

- El paciente "predice" su empeoramiento o su falta de mejoría.

- Al menos, un profesional de la salud implicado en el diagnóstico y tratamiento sospecha de la posibilidad de simulación. 
Se puede sospechar falta de consistencia interna en las pruebas cuando aparece alguna de las siguientes anormalidades:

- Memoria de reconocimiento exageradamente alterada.

- Reducido efecto de primacía en las pruebas de evocación libre de material aprendido.

- Puntuaciones de atención y concentración muy inferiores a las de memoria general.

- Mejor recuerdo de los ítems difíciles que de los fáciles.

- Discrepancia entre los resultados de tests que evalúan un mismo dominio neurocognitivo.

- Menor puntuación que enfermos emparejados por comportamiento en situaciones no estructuradas.

- Rendimiento final por debajo del esperable por azar.

- No se pueden duplicar las ejecuciones en la misma prueba en sesiones separadas.

- Ocurrencia de respuestas absurdas o ilógicas.

- Ningún efecto de las claves en las tareas de recuerdo retardado.

- Un patrón de ejecuciones malo-bueno-malo en evaluaciones neuropsicológicas seriadas, efecto de monotonía o un significativo mayor número de falsas respuestas.

Los Índices de Sospecha de Simulación de Resnick, Hall \& Pritchard son:

- Mantiene la capacidad de esparcimiento.

- Historial laboral desfavorable.

- Evasivo, resentido, susceptible o poco dispuesto en la entrevista.

- Intenta evitar las pruebas más objetivas.

- Discurso sin fisuras, muy elaborado, estructurado y controlado.

- Emociones inexpresivas o exageradas, prolongadas en el tiempo y con inicio/ decremento bruscos (Tr. Ideas delirantes).

- Sintomatología contradictoria con respuestas evasivas (no recuerdo, depende, etc.) ante preguntas dirigidas.

- Sintomatología estrafalaria.

- Evaluar todas las áreas: laboral, familiar, social, recreativa, etc.:

- Baja laboral pero...

- No existe búsqueda de soluciones.

- Sobreactuación (ve menos que el ciego, oye menos que el sordo y...).

Se pueden establecer las siguientes directrices generales para la detección:

- Descartar psicopatología. El cuadro no encaja en la nosología.

- Descartar Trastornos Facticios con síntomas psicológicos.

- Detectar factores de riesgo de simulación-metasimulación:

- Incentivo externo: obligaciones, beneficios legales, beneficios económicos, etc.

- Personalidad antisocial.

- Detectar signos de sospecha (test, etc.).

- Abordaje directo por confrontación o confesión.

Como ya se ha adelantado, la detección de la simulación se fundamenta en la idea central de la convergencia de los datos que provienen de distintas fuentes de información.

Cuantas más inconsistencias presente un paciente a lo largo de la realización de diferentes pruebas independientes y en diferentes dimensiones, más plausible resultará 
pensar que su rendimiento refleja un esfuerzo deliberado por dar una imagen falsa de sus capacidades o sintomatología.

Esta evaluación debe realizarse desde un enfoque multidisciplinar, teniendo en cuenta la información que puedan aportar los distintos profesionales implicados en el tratamiento del paciente.

La convergencia de datos provenientes de diferentes fuentes de información es crucial para asegurar el diagnóstico de simulación.

Si tras la realización de una historia clínica completa, entrevista y exploración física y psicológica, el profesional encuentra inconsistencias que le hacen sospechar simulación, debe realizar pruebas de screening de simulación (médicas, autoinformes, psicofisiológicas, neuropsicológicas, observacionales, etc.), y, dependiendo de la convergencia de los datos encontrados, se podrá llegar o no al diagnóstico de simulación.

Existe un buen número de pistas o signos que se pueden buscar en la detección del engaño como evidencia objetiva de la simulación:

- Retención de información y Falta de cooperación.

- Exageración.

- Ansiedad por llamar la atención.

- Conductas extrañas.

- Ausencia de alteración afectiva clínicamente asociada con la enfermedad que simula.

- Ausencia de perseveración.

- Incoherencia entre resultados de pruebas y funcionalidad del evaluado.

- Alteraciones del lenguaje no verbal.

- Síntomas generalmente incongruentes con los síntomas legítimos de la enfermedad mental.

- La simulación es más difícil de mantener por períodos largos.

- Dificultad para fingir síntomas fisiológicos.

- Simulan síntomas evidentes y en relación con el conocimiento previo de la enfermedad.

- Se fingen más síntomas que cuadros diagnósticos íntegros.

- Informan síntomas severos con aparición aguda en contradicción con el desarrollo crónico conocido por los clínicos.

- Discrepancia entre el auto-reporte y los archivos médicos.

- Los simuladores parecen no tener ningún motivo o comportamiento psicótico asociado a sus ofensas.

- Evidencia de complicidad.

- Engaños anteriores.

- Historia laboral incoherente con la enfermedad.

En nuestro contexto se puede señalar la influencia de diversos factores en la presencia y extensión en la población de la simulación-disimulación de enfermedades y contingencias, así como del retraso en la reincorporación al trabajo tras una baja laboral y, según García Caro et al. ${ }^{8}$, éstos pueden ser:

- Transferencia del control clínico de las bajas laborales del médico de familia al especialista, por un rechazo subconsciente de la responsabilidad de las mismas, originando retraso en la toma de decisión.

- La deshumanización de la medicina y la desorganización del sistema sanitario, muy imbricadas entre sí. 
- Diferencia de criterios entre los distintos facultativos que atienden al paciente (papel terapéutico de la reincorporación al trabajo).

- Falta de concienciación de la sociedad, en general, y de cada uno, en particular, del gasto irracional que suponen las bajas laborales y las incapacidades permanentes no suficientemente justificadas.

- Escasa o nula participación de los trabajadores sociales en la labor de preparación del nicho familiar, social y laboral adecuados.

- Malas relaciones laborales, que obstaculizan la reincorporación al trabajo.

- Resistencia por parte del mismo interesado a su reintegración laboral (considerarse no bien recuperado, temer una posible recaída).

- Actitud similar por parte de sus familiares.

- Oposición de las empresas y de los médicos de empresa (s. de prevención) a la vuelta al trabajo de cierto tipo de enfermos por los posibles problemas que pudieran plantear.

Por último, en la actualidad, una serie de factores debidos a la situación económica y crisis laboral (crisis de las empresas, reconversiones, regulaciones de plantilla y/o empleo, situación del mercado laboral).

\section{DIAGNÓSTICO DE LA SIMULACIÓN Y DISIMULACIÓN}

En algunos casos, el diagnóstico de la patomimia puede ser de muy fácil resolución, mientras que en otros puede entrañar una gran dificultad.

De cualquier forma, en todos estos casos falta de manera sistemática la sinceridad y la confianza del paciente hacia el médico evaluador (y muchas veces, de forma inconsciente, al revés).

Lo primero es puntualizar que durante la exploración y el estudio del paciente en el contexto evaluador hay que tener en cuenta las siguientes recomendaciones:

- Nunca hay que dar la impresión de que se sospecha una simulación/disimulación.

- Las maniobras deben efectuarse sin vacilación en medio de otras fases de la exploración clínica.

- No deben ser observadas por acompañantes.

- Los detalles de estas maniobras no deben divulgarse en nuestro informe, que debería ceñirse a precisar que se han llevado a cabo "métodos objetivos de control" si fuera necesario.

- El diagnóstico de una simulación conlleva siempre dos operaciones diagnósticas: en primer lugar, el diferenciar una simulación de una patología orgánica o psíquica verdadera y, en segundo lugar, aclarar si tras la fachada de una simulación se esconde una enfermedad mental o es simplemente simulación.

Según J.M. Vicente Pardo, existen una serie de signos que pueden ayudarnos en la detección de una posible simulación, como son el comportamiento durante la evaluación, las características de los síntomas simulados y ciertos aspectos de la historia clínica.

Marco et al. distinguieron en 1990, entre otros, los siguientes tipos de simulación ${ }^{2,9}$ :

- Verdadera o total. El sujeto finge conscientemente algo inexistente.

- Sobresimulación o exageración de los síntomas que existen realmente, o sobre los que existen se suman otros inexistentes.

- Metasimulación o perseveración. Prolongar o revivir síntomas de un trastorno verdadero del que curó recientemente o padeció en épocas anteriores, estando ahora sano. 
- Presimulación. Simulación realizada antes de un hecho con la intención de invocarla después.

- Simulación invertida o disimulación.

Las diversas formas de producirla que se han descrito están muy relacionadas con estos distintos tipos:

- Creatriz: se crea algo que no existe.

- Evocatriz: se evocan síntomas que se padecieron con anterioridad, pero no actualmente.

- Imitatriz: imita gestos o síntomas que ha visto en otros.

- Exageratriz: los síntomas existentes, leves, son magnificados, exagerados.

- Perseveratriz: se prolonga indebidamente el estado evolutivo, la duración.

Los medios que podemos y debemos emplear en el diagnóstico de la simulacióndisimulación son:

- Examen médico o psiquiátrico completo.

- Comprobación de los hallazgos:

- a) por el estudio de la documentación médica, especialmente el seguimiento evolutivo reflejado en la historia clínica,

- y b) por el estudio de las pruebas complementarias.

\section{Examen médico}

Se realizará una historia clínica adecuada y dirigida, con un interrogatorio concienzudo, intentando atraernos la confianza del entrevistado. Hay que valorar los datos con prudencia y precaución, evitando actitudes de susceptibilidad. Además, se debe recordar que la ciencia médica no es exacta, y que cabe la posibilidad de que síntomas aislados o anómalos puedan deberse a evoluciones extrañas de un proceso patológico real. Por todo ello, hay que ser cauteloso e intentar no hacer un juicio con la primera impresión, sino que conviene estudiar al enfermo en profundidad.

Antes de diagnosticar una simulación debe realizarse siempre una completa valoración médica ${ }^{6}$.

Para ello, además del examen clínico o psiquiátrico, es siempre recomendable el uso de las técnicas complementarias adecuadas en función del cuadro que presente el sujeto (bioquímicas, radiológicas, electroencefalograma, ecocardiograma, resonancia magnética nuclear, etc.).

Desgraciadamente, no existe un signo patognomónico de la simulación, porque además de los criterios que señalábamos anteriormente, algunas circunstancias y el sentido común deben inducirnos a la sospecha en las lesiones provocadas y/o patologías "incongruentes".

Además de las pruebas complementarias objetivas, los procedimientos que pueden usarse para desenmascarar la patología simulada, y que pueden considerarse correctos éticamente, son los “procedimientos de sorpresa”, que tienen por objeto coger desprevenido al sujeto y poner de manifiesto la falsedad del síntoma alegado por el mismo.

\section{Exploración psicológico-psiquiátrica}

Tanto si hay sólo simulación de enfermedades físicas, como si se trata de evidenciar la simulación de enfermedades mentales, es necesaria la realización de un examen psicológico-psiquiátrico. El diagnóstico de la simulación exige el conocimiento profundo de la etiología, los síntomas físicos y psíquicos de los distintos cuadros psicopatológicos.

Al igual que de modo general, se han señalado diversos índices que orientan hacia el diagnóstico de la simulación de la enfermedad mental: 
- El cuadro clínico se manifiesta por primera vez en un contexto judicial o administrativo.

- El síndrome simulado tiene caracteres atípicos, no se ajusta a los del síndrome clínico verdadero, presenta combinaciones de manifestaciones de diversos síndromes, hay discrepancia entre los síntomas alegados por el sujeto y los hallazgos objetivos detectados.

- El verdadero enfermo mental ha perdido la noción de enfermedad mental; el simulador manifiesta que es un enfermo psíquico.

- Son frecuentes las respuestas evasivas (movimientos, palabras o frases estereotipadas).

- Mala predisposición para el interrogatorio y la evaluación diagnóstica.

- Falta de estabilidad y continuidad del cuadro clínico (el simulador lo va adaptando a las exigencias de las dudas que va planteando el médico).

- Falta de correlación entre la sintomatología simulada y las causas etiológicas más frecuentes.

- Suelen presentar trastornos antisociales de la personalidad.

Clásicamente se recomendaba el empleo de métodos indirectos para el diagnóstico de la simulación destinados a hacer confesar al simulador su fraude, pero la Psicología y la Psiquiatría modernas han desarrollado técnicas que son capaces de revelar los fenómenos que ocurren en el subconsciente y que escapan al control de la voluntad.

\section{MODALIDADES DE SIMULACIÓN}

Cada tipo de prestación de incapacidad en la Seguridad Social, siguiendo en parte a J.J. Álvarez Sáenz ${ }^{5}$, tiene diversas modalidades, cada una con unas características de simulación diferentes.

En la incapacidad temporal (IT) podemos encontrar:

- Simulación o exageración de sintomatología con intención de conseguir el parte de baja.

- Una vez obtenida, con o sin motivación clínica, se sigue refiriendo persistencia de clínica con intención de mantenerla.

- Búsqueda de IT en la proximidad de la finalización de un contrato, o inmediatamente antes de concluir la percepción de las prestaciones por desempleo.

- Simultanear la situación de IT con una actividad laboral.

- Personas que no tienen carencia (cotizaciones suficientes para optar a una incapacidad permanente) y buscan/alargan procesos de IT para intentar conseguirla.

Y en la incapacidad permanente (IP) podemos detectar:

- Suplantación o sustitución (simulación) de persona, parecidos (hermanos, gemelos, etc.) o no. Se soluciona con el requerimiento, en todo reconocimiento tanto en IT como IP, de que se acredite la identidad, mediante DNI o tarjeta de residencia, pasaporte o permiso de conducir, vigentes.

- Simulación de la profesión. Referirá profesiones generales, sin especificar puesto de trabajo. Alegará trabajos con riesgo, de esfuerzo físico o psíquico intenso. Suele citar como profesión aquélla que más tiempo ha realizado en su vida laboral, y no la que realizaba al iniciar el proceso de IT/IP. Pueden existir cambios (recalificaciones) profesionales falsas durante la reestructuración de plantilla, etc.

- Falsificación de pruebas médicas. De otras personas, o manipulación/falsificación de informes. 
- Informes médicos de complacencia.

- Alta de afiliación con una edad avanzada, con antecedentes de patologías incapacitantes.

- Alta de afiliación en determinadas actividades profesionales, con posible calificación de enfermedad profesional que realiza una disimulación.

También podemos encontrar distintos ejemplos según la forma en que se intenta simular la contingencia del proceso, por el beneficio que conllevan las contingencias profesionales:

- Alteración y simulación de la cronopatología del suceso. El paciente altera la fecha y hora del comienzo de la clínica para que sea accidente de trabajo, bien en lugar de accidente no laboral, o bien en lugar de enfermedad común.

- Alteración y simulación en la confección del parte de accidente, con acuerdo entre el trabajador y la empresa, alterando las circunstancias del mismo, el horario y la fecha en que ocurrió el suceso, etc., bien con objeto de obtener una mejora de las condiciones para el trabajador, bien para ocultar alguna negligencia de falta de medidas de seguridad e higiene.

\section{BIBLIOGRAFÍA}

* Chavarría Puyol, H. M., Díaz Salazar, C. E. Reflexiones sobre la simulación y disimulación en medicina evaluadora. Rehabilitación. 2008; 42 (6):325-30.

1. Estarellas Roca, A.M. Simulación en la valoración del daño corporal. Cuad Med For. 1997;10:21-8.

2. Presentación Blasco, J.A., et al. La simulación en psiquiatría forense: instrumentos para su detección. Comunicacions Lliures Psiquiatría Forense. Sisenes Jornades Catalanes D'Actualització en medicina forense. Barcelona, Noviembre de 2001.

3. Gisbert Calabuig, J., Villanueva Cañadas, E. Medicina legal y toxicología. 6. ${ }^{a}$ ed. Barcelona: Masson S.A.; 2004. p. 1394.

4. Quezada-Ortega, M. R., et al. Simulación en trabajadores que solicitan pensión por invalidez laboral. Gac Méd Méx. 2006;142(2):109-12.

5. Álvarez Sáenz, J. J. La simulación y disimulación. Incidencia en el marco laboral de la legislación española. Diagnóstico en VDC. 2001;13:12-5.

6. Inda Caro, M., et al. La simulación de enfermedad física o trastorno mental. Papeles del Psicólogo. 2005;26:99-108.

7. Figuerido Poulain, J. L. Engaño, enfermedades autoinfligidas y simulación. Salud Mental Atención Primaria. Salud Global. Año I, Número 1, 2001, p.1-7.

8. García Caro, A. L., et al. La valoración médica de la invalidez laboral por daño psíquico. Cuad Med For. 1997;8:60-8.

9. Mélennec, L. Valoración de las discapacidades y del daño corporal. París: Masson Editeur; 1991.

* No existe conflicto de intereses. 